Proyecciones

Vol. 18, ㄲo 2, pp. 155-164, December 1999

Universidad Católica del Norte

Antofagasta - Chile

\title{
SOLUTIONS TO THE PRESCRIBED MEAN CURVATURE EQUATION
}

\author{
PABLO AMSTER \\ P. DE NÁPOLI \\ and \\ M. C. MARIANI \\ Universidad de Buenos Aires - Argentina
}

\begin{abstract}
We apply variational methods in order to prove that the nonlinear system (1) admits at least one regular solution.
\end{abstract}




\section{Introduction}

We consider the problem of finding $u \in H^{1}\left(\Omega, \mathbf{R}^{3}\right)$ such that

$$
\left\{\begin{array}{l}
\Delta u=2 H(u) u_{x} \wedge u_{y} \text { in } \Omega \\
u=\gamma \text { in } \partial \Omega
\end{array}\right.
$$

where $H$ is a given continuously differentiable function and $\Omega$ is an open subset of $\mathbf{R}^{2}$ with $C^{1}$ boundary.

The system of differential equations above is the Dirichlet problem for the prescribed mean curvature equation in isothermal coordinates, also called H-system, which arises in the generalized Plateau's problem (see $[\mathrm{O}]$ ).

Hildebrant, Wente (see [W1,W2]) and others have studied the problem for constant $H$. For this case, Brezis-Coron and Struwe have shown by variational methods (see $[\mathrm{BC}],[\mathrm{S}]$ ) that if the boundary data is small and non constant, then there are at least two weak solutions. The problem has been also studied by fixed point methods (see [AMR]).

The goal of this work is to show that also for non constant $H$, the problem may be solved by variational methods, under rather general conditions on $H$ and the boundary data.

Our main result is the following:

Theorem $1:$ Let $\gamma \in H^{1 / 2}\left(\partial \Omega, \mathbf{R}^{3}\right) \cap L^{\infty}\left(\partial \Omega, \mathbf{R}^{3}\right), R=\|\gamma-c\|_{L^{\infty}\left(\partial \Omega, \mathbf{R}^{3}\right)}$ for some constant $c$, and $B \subset \mathbf{R}^{3}$ the closed ball of radius $\mathbf{R}$

centered at $c$. Then, if $H \in C^{1}(B)$ satisfies

$$
\|H\|_{L^{\infty}(B)}<\frac{1}{R}
$$

then problem (1) admits a weak solution $u \in H^{1}\left(\Omega, \mathbf{R}^{3}\right) \cap L^{\infty}\left(\Omega, \mathbf{R}^{3}\right)$, with $\|u-c\|_{L^{\infty}(B)} \leq R$.

Remark : By a result of Bethuel ([B]) weak solutions of the mean curvature equation are $C^{2, \alpha}(\Omega)$ for $H$ bounded and Lipschitz continuous. Moreover, by a result of Chanillo and $\mathrm{Li}([\mathrm{CL}])$ the solutions are continuous up to the boundary. 


\section{Variational formulation of the problem}

For simplicity, we may assume that $c=0$ and extend $H$ to a function of class $C^{1}\left(\mathbf{R}^{3}\right)$ such that $\|H\|_{L^{\infty}\left(\mathbf{R}^{3}\right)}<\frac{1}{R}$.

We define the vector field $q: \mathbf{R}^{3} \rightarrow \mathbf{R}^{3}$ :

$$
q(x, y, z)=\frac{4}{3}\left(\int_{0}^{x} H(t, y, z) d t, \int_{0}^{y} H(x, t, z) d t, \int_{0}^{z} H(x, y, t) d t\right)
$$

It is immediate that $\operatorname{div} q=4 H$ and $|q(x, y, z)| \leq \frac{4}{3}\|H\|_{L^{\infty}}|(x, y, z)|$.

We'll work in the Banach space $H^{1}\left(\Omega, \mathbf{R}^{3}\right) \cap L^{\infty}\left(\Omega, \mathbf{R}^{3}\right)$, with the norm:

$$
\|u\|=\|u\|_{H^{1}}+\|u\|_{L^{\infty}}
$$

and consider energy functional

$$
E(u)=D(u)+Q(u)
$$

where $D$ is the Dirichlet's integral and $Q$ is the functional

$$
Q(u)=\int_{\Omega} q(u) \cdot\left(u_{x} \wedge u_{y}\right)
$$

We'll use the estimatives

$$
\begin{gathered}
\left.|Q(u)| \leq \int_{\Omega}|q(u)| \cdot \mid u_{x} \wedge u_{y}\right)\left.\left|\leq \frac{2}{3}\|H\|_{L^{\infty}}\|u\|_{L^{\infty}} \int_{\Omega}\right| \nabla u\right|^{2} \\
E(u) \geq\left(1-\frac{2}{3}\|H\|_{L^{\infty}}\|u\|_{L^{\infty}}\right) \int_{\Omega}|\nabla u|^{2}
\end{gathered}
$$

\section{Technical lemmas}

In this section we present some technical facts.

Lemma $2 Q$ is continuous in $H^{1} \cap L^{\infty}$.

Lemma 3 We consider the functional

$$
I(u, h)=\int_{\Omega} H(u) \cdot\left(u_{x} \wedge u_{y}\right) \cdot h
$$


where $u \in H^{1} \cap L^{\infty}$ and $h \in L^{\infty}$.

Then $I$ is continuous.

\section{Proof :}

Lemma 2 and Lemma 3 follow from simple computations.

\section{Lemma 4}

The functional $E$ is Gateaux differentiable in $H^{1} \cap L^{\infty}$ for variations in $H_{0}^{1} \cap L^{\infty}$, and

$$
d E(u)(h)=2 \int_{\Omega} \nabla u \cdot \nabla h+\int_{\Omega} 4 H(u) \cdot\left(u_{x} \wedge u_{y}\right) \cdot h
$$

for every $u \in H^{1} \cap L^{\infty}, h \in H_{0}^{1} \cap L^{\infty}$.

\section{Proof :}

Let us first take $u, h \in C^{1}$ and define a function $U: \Omega \times \mathbf{R} \rightarrow \mathbf{R}^{3}$ by:

$$
U(x, y, t)=u(x, y)+t \cdot h(x, y)
$$

We'll write $u^{t}(x, y)=U(x, y, t)$.

We recall that the field $q$ in $\mathbf{R}^{3}$ is associated to the 2 -form $\omega$ given by:

$$
\omega(X, Y)=(X \wedge Y) \cdot q(u)
$$

with

$$
d \omega=\operatorname{div}(q) d u^{1} \wedge d u^{2} \wedge d u^{3}
$$

Applying Stokes theorem to the cylinder $C=\Omega \times[0, \varepsilon]$, we obtain:

$$
\int_{C} d\left(U^{*} \omega\right)=\int_{\partial C} U^{*} \omega
$$

Moreover,

$$
\begin{gathered}
d\left(U^{*} \omega\right)=U^{*}(d \omega)=U^{*}\left(\operatorname{div} q(u) d u^{1} \wedge d u^{2} \wedge d u^{3}\right) \\
=4 H(U) \cdot \frac{\partial\left(U^{1}, U^{2}, U^{3}\right)}{\partial(x, y, t)} d x \wedge d y \wedge d t=4 H\left(u^{t}\right) \cdot\left(u_{x}^{t} \wedge u_{y}^{t}\right) \cdot h
\end{gathered}
$$


We have:

$$
U^{*} \omega=\omega\left(\frac{\partial U}{\partial x}, \frac{\partial U}{\partial y}\right) d x \wedge d y=q\left(u^{t}\right) \cdot\left(u_{x}^{t} \wedge u_{y}^{t}\right)
$$

on $\Omega \times\{\varepsilon\}$ and $\Omega \times\{0\}$ and

$$
U^{*} \omega=\omega\left(\frac{\partial U}{\partial \sigma}, \frac{\partial U}{\partial t}\right) d \sigma \wedge d t=q\left(u^{t}\right) \cdot\left(\frac{\partial U}{\partial \sigma} \wedge h\right) d \sigma \wedge d t
$$

on the lateral surface $\partial \Omega \times[0, \varepsilon]$, where $\sigma$ is the unit tangent vector on $\partial \sigma$.

Thus,

$$
\begin{gathered}
\int_{0}^{\varepsilon} \int_{\Omega} 4 H\left(u^{t}\right) \cdot\left(u_{x}^{t} \wedge u_{y}^{t}\right) \cdot h d x \wedge d y \wedge d t= \\
\int_{\Omega \times\{\varepsilon\}} q\left(u^{\varepsilon}\right) \cdot\left(u_{x}^{\varepsilon} \wedge u_{y}^{\varepsilon}\right) d x \wedge d y \wedge d t-\int_{\Omega \times\{0\}} q(u) \wedge\left(u_{x} \wedge u_{y}\right) \cdot h \\
+\int_{\partial \Omega \times[0, \varepsilon]} q(u) \cdot\left(\frac{\partial U}{\partial \sigma} \wedge h\right) d \sigma \wedge d t
\end{gathered}
$$

Assuming that $h=0$ on the boundary of $\Omega$, we get:

$$
\int_{0}^{\varepsilon} \int_{\Omega} 4 H\left(u^{t}\right) \cdot\left(u_{x}^{t} \wedge u_{y}^{t}\right) \cdot h=Q\left(u^{\varepsilon}\right)-Q(u)
$$

By density, this formula holds for $u \in H^{1} \cap L^{\infty}$ and $h \in H_{0}^{1} \cap L^{\infty}$.

By dominated convergence we conclude that

$$
d Q(u)(h)=\int_{\Omega} 4 H(u) \cdot\left(u_{x} \wedge u_{y}\right) \cdot h
$$

which completes the proof.

Remark: By lemmas 2 and $3 d E$ is continuous, i.e. $E \in C^{1}\left(H_{0}^{1} \cap L^{\infty}\right)$.

\section{Corollary 5 :}

If $u$ is a critical point of functional $E$ for variations in $H_{0}^{1} \cap L^{\infty}$ then $u$ is a weak solution of (1). 


\section{Proof of Theorem 1}

We fix $R^{\prime}>R$ such that $\|H\|_{L^{\infty}} R^{\prime}<1$ and consider the nonempty set:

$$
K=\left\{u \in H^{1}\left(\Omega, \mathbf{R}^{3}\right): u=\gamma \text { in } \partial \Omega,\|u\| \leq R^{\prime}\right\}
$$

Then we obtain

\section{Lemma 6 :}

$E$ achieves a minimum in $K$.

\section{Proof :}

By (3), the functional $E$ is bounded from below in $K$. Let $E_{0}=$ $\inf _{v \in K} E(v)$ and consider a minimizing sequence $\left(u^{n}\right)$

By (3) and Poincaré's inequality $u^{n}$ is bounded in $H^{1}$, and then we may suppose that

$$
u^{n} \rightarrow \underline{u} \text { in } H^{1} \text { weakly }
$$

and

$$
u^{n} \rightarrow \underline{u} \text { a.e. }
$$

By the continuity of $q$ we conclude that:

$$
q\left(u^{n}\right) \rightarrow q(\underline{u}) \text { a.e. }
$$

and since $\left|q\left(u^{n}\right)\right| \leq \frac{4}{3}\|H\|_{L^{\infty}}$, we may also assume that:

$$
q\left(u^{n}\right) \rightarrow q(\underline{u}) \text { in } L^{\infty} \text { weak }^{*}
$$

Set $\theta^{n}=u^{n}-u \in H_{0}^{1}$. Then $\left\|\theta^{n}\right\|_{L^{\infty}} \leq 2 R^{\prime}$, and

$$
\begin{gathered}
E\left(u^{n}\right)=\int_{\Omega}|\nabla \underline{u}|^{2}+2 \int_{\Omega} \nabla u \cdot \nabla \theta^{n} \\
+\int_{\Omega}\left|\nabla \theta^{n}\right|^{2}+\int_{\Omega} q\left(u^{n}\right) \cdot\left(\underline{u}_{x}+\theta_{x}^{n}\right) \wedge\left(\underline{u}_{y}+\theta_{y}^{n}\right)
\end{gathered}
$$

Thus,

$$
\int_{\Omega} \nabla u \cdot \nabla \theta^{n} \rightarrow 0
$$


Moreover, being

$$
\int_{\Omega} q\left(u^{n}\right) \cdot\left(\underline{u}_{x} \wedge \theta_{y}^{n}\right)=-\int_{\Omega} \theta_{y}^{n} \cdot\left(q\left(u^{n}\right) \wedge \underline{u}_{x}\right)
$$

then

$$
\int_{\Omega} q\left(u^{n}\right) \cdot\left(\underline{u}_{x} \wedge \theta_{y}^{n}\right) \rightarrow 0,
$$

since $\theta_{y}^{n} \rightarrow 0$ weakly in $L^{2}$, and

$$
\left|q\left(u^{n}\right) \wedge \underline{u}_{x}\right|^{2} \leq\left(\frac{4}{3}\left|u^{n}\right|\right)^{2} \cdot\left|\underline{u}_{x}\right|^{2} \leq\left(\frac{4}{3} R^{\prime}\right)\left|\underline{u}_{x}\right|^{2} .
$$

In the same way, we conclude that

$$
\int_{\Omega} q\left(u^{n}\right) \cdot\left(\theta_{x}^{n} \wedge \underline{u}_{y}\right) \rightarrow 0
$$

We also have the estimative:

$$
\begin{gathered}
\left.\left|\int_{\Omega} q\left(u^{n}\right) \cdot \theta_{x}^{n} \wedge \theta_{y}^{n}\right| \leq \int_{\Omega} 4\|H\|_{L^{\infty}} \| u^{n}\right) \|_{L^{\infty}}\left|\theta_{x}^{n} \wedge \theta_{y}^{n}\right| \\
\leq \frac{2}{3}\|H\|_{L^{\infty}} R^{\prime} \int_{\Omega}\left|\nabla \theta^{n}\right|^{2} \leq \frac{2}{3} \int_{\Omega}\left|\nabla \theta^{n}\right|^{2}
\end{gathered}
$$

Finally as $q\left(u^{n}\right) \rightarrow q(\underline{u})$ weak* in $L^{\infty}$ :

$$
\int_{\Omega} q\left(u^{n}\right) \cdot\left(\underline{u}_{x} \wedge \underline{u}_{y}\right)=\int_{\Omega} q(\underline{u}) \cdot\left(\underline{u}_{x} \wedge \underline{u}_{y}\right)+o(1)
$$

Then,

$$
E\left(u^{n}\right) \geq E(u)+o(1)+\frac{1}{3} \int_{\Omega}\left|\nabla \theta^{n}\right|^{2}
$$

and we can conclude that $E(u)=E_{0}$ and $\int_{\Omega}\left|\nabla \theta^{n}\right|^{2} \rightarrow 0$.

\section{Lemma 7 :}

Let $u \in K$ satisfy $E(u)=\inf _{v \in K} E(v)$. Then $u$ is a weak solution of (1).

\section{Proof :}

Let us fix a nonnegative test function $\eta \in \mathcal{D}(\Omega)$. Then $u(1-\varepsilon \eta) \in K$ for $\varepsilon \geq 0$ small enough. 
As the function $\varphi(\varepsilon)=E(u(1-\varepsilon \cdot \eta))$ has a minimum in $\varepsilon=0$, then $\varphi^{\prime}(0)=d E(u)(-u \cdot \eta) \geq 0$, i.e.

$$
2 \int_{\Omega} \nabla u \cdot \nabla(-\eta \cdot u)+\int_{\Omega} 4 H(u)\left(u_{x} \wedge u_{y}\right) \cdot(-\eta u) \geq 0 \forall \eta \in \mathcal{D}(\Omega)
$$

By Green's formula,

$$
\int_{\Omega} \triangle\left(|u|^{2}\right) \cdot \eta=-\int_{\Omega} \nabla\left(|u|^{2}\right) \nabla(\eta)=-\int_{\Omega} 2(\nabla u \cdot u) \cdot \nabla \eta
$$

It follows that

$$
-\frac{1}{2} \triangle|u|^{2}+|\nabla u|^{2}+2 H(u) u \cdot u_{x} \wedge u_{y} \leq 0 \text { in } \mathcal{D}^{\prime}(\Omega)
$$

Then, as

$$
\left|2 H(u) u \cdot\left(u_{x} \wedge u_{y}\right)\right| \leq\|H\|_{L^{\infty}} R^{\prime}|\nabla u|^{2}
$$

it follows that

$$
-\triangle|u|^{2} \leq 0 \text { in } \mathcal{D}^{\prime}(\Omega)
$$

and then by Stampacchia's maximum principle (see [GT]) we get:

$$
\sup _{\Omega}|u|=\sup _{\partial \Omega}|u|=R
$$

Since $R<R^{\prime}, u$ is interior in $K$. It follows that $d E(u)(v)=0$ for any $v \in H_{0}^{1} \cap L^{\infty}$, and by corollary $5 u$ is a weak solution of (1). 


\section{References}

[1] Anster P. Mariani, M. C., Rial, D. F. : Existence and uniqueness of H-System's solutions with Dirichlet conditions. To appear in Nonlinear Analysis, Theory, Methods, and Applications.

[2] Bethuel, F. : Un résultat de regularité pour les solutions de l'equation des surfaces à courboure moyenne prescrite. R. Acad. Sci. Paris Sér. I Math 314, $n^{\circ} 13$, pp. $1003-100$, (1992).

[3] Brezis, H., Coron, J., Multiple Solutions of H-Systems and Rellich's conjecture. Comm. Pure Appl. Math 37, pp. 149 - 187, (1984).

[4] Chanillo S., Li Y. : Continuity of Solutions of Uniformly Elliptic Equations in $\mathbf{R}^{2}$. Manulscripta math. 77, pp. 415-433, (1992).

[5] Gilbarg, D., Trudinger N. : Elliptic Partial Differential Equations of Second Order. Springer-Verlag (1977).

[6] Osserman, R. : A Survey of Minimal Surfaces. Van Nostrand Reinhold Company, (1969).

[7] Struwe, M.: Variational Methods, Applications to Nonlinear Partial Differential Equations and Hamiltonian Systems, Springer-Verlag, pp. 180.

[8] Wente, H. : An Existence Theorem for Surfaces of Constant Mean Curvature. Journal of Mathematical Analysis and Applications 26, pp. 318-344, (1969)

[9] Wente, $H$. : The differential equation $\Delta X=2 H\left(X_{u} \wedge X_{v}\right)$ with vanishing boundary values. Proceedings of the American Mathematical Society 50, pp.131-7, (1975).

Received : December, 1998 


\section{P.Amster \\ P. De Nápoli \\ and \\ M. C. Mariani}

Departamento de Matemática

Fac. de Cs. Exactas y Naturales

Universidad de Buenos Aires

Consejo Nacional de Investigaciones Científicas y Técnicas (CONICET)

Pab. I, Ciudad Universitaria (1428)

Buenos Aires

Argentina 